Temperature-dependent efficiency droop in InGaN-based light-emitting diodes induced by current crowding

This article has been downloaded from IOPscience. Please scroll down to see the full text article.

2012 Semicond. Sci. Technol. 27055013

(http://iopscience.iop.org/0268-1242/27/5/055013)

View the table of contents for this issue, or go to the journal homepage for more

Download details:

IP Address: 194.44.60.2

The article was downloaded on 04/04/2012 at 10:23

Please note that terms and conditions apply. 


\title{
Temperature-dependent efficiency droop in InGaN-based light-emitting diodes induced by current crowding
}

\author{
Ya Ya Kudryk ${ }^{1}$, A K Tkachenko ${ }^{2}$ and A V Zinovchuk ${ }^{2}$ \\ ${ }^{1}$ V. Lashkaryov Institute of Semiconductor Physics, 03028 Kyiv, Ukraine \\ ${ }^{2}$ Ivan Franko Zhytomyr State University, 10008 Zhytomyr, Ukraine \\ E-mail: zinovchuk.a@zu.edu.ua
}

Received 11 January 2012, in final form 10 February 2012

Published 3 April 2012

Online at stacks.iop.org/SST/27/055013

\begin{abstract}
Temperature-dependent internal quantum efficiency (IQE) of multiple quantum well $\mathrm{InGaN} / \mathrm{GaN}$ light-emitting diodes (LEDs) has been investigated. IQE versus current relation is analysed using the modified rate equation model that takes into account the current crowding effect at different temperatures. The results of calculations are consistent with the fact that droop in IQE at higher currents originates from Auger recombination increased by current crowding. It is shown that unusual experimentally observed temperature dependence of the efficiency droop can be explained by stronger lateral nonuniformity of carrier injection at low temperatures without any assumptions about carrier delocalization from In-rich regions in quantum wells.
\end{abstract}

(Some figures may appear in colour only in the online journal)

\section{Introduction}

The nonthermal reduction of the internal quantum efficiency (IQE) with current increasing is the well-known fundamental 'efficiency droop' problem for InGaN-based LEDs. Strong droop behaviour limits the development of many applications that require the operation of the InGaN LEDs under high injection levels. The efficiency of available multiple quantum well (MQW) InGaN/GaN LEDs has a maximum at current densities (10-20 $\mathrm{A} \mathrm{cm}^{-2}$ ) that are significantly lower than currents necessary for application in general illumination $\left(>200 \mathrm{~A} \mathrm{~cm}^{-2}\right)$ [1]. Thus, reduction of the efficiency droop is one of the most important issues that must be overcome for the future progress of LED lighting. Since the strong efficiency droop is not observed in GaAs and GaP-based LEDs, the origin of this phenomenon is not fully understood and still controversial. Different mechanisms have been suggested to account for the efficiency droop in InGaN LEDs: Auger recombination [1], carrier delocalization from In-rich regions [2,3], leakage processes caused by spontaneous and piezoelectric polarization fields in QWs $[4,5]$.

Recently, unusual temperature dependence of the efficiency versus current relation for blue and green devices has been experimentally observed [6, 7]. At lower temperatures droop is more pronounced and IQE reaches its peak value at lower currents. These experimental results can be explained in terms of theoretical models that include carrier delocalization from In-rich regions in conjunction with nonradiative recombination at threading dislocations [8], saturation of radiative recombination rate [9] or saturation of the available localized states within the quantum well [10]. It is believed that due to the decrease of the Auger coefficient at lower temperatures, the Auger recombination mechanism is not capable of explaining temperature behaviour of the droop. However, in [11] we found that lateral nonuniformity of the carrier injection in the active layer provoked by the current crowding (CC) effect causes the substantial increase of Auger recombination rate and makes the efficiency droop increase. Destructive influence of the CC on the InGaN LED efficiency was also obtained in [12]. The CC is a wellestablished effect in large-area InGaN LEDs on insulating sapphire substrates which causes localization of remarkable current flow routes near the edges of $\mathrm{p}$ - or n-type contacts and lateral nonuniformity of light emission [13]. In this paper, based on the model reported in [11], we have shown that Auger recombination increased by strong $\mathrm{CC}$ at lower 
temperatures may be an alternative mechanism which controls the temperature dependence of the IQE and its droop in InGaN LEDs.

\section{Simulation procedure}

The first stage of the simulation procedure includes the finite element calculation of the current density distributions inside LED at different applied biases. The simulations were performed for the simplified structure of the $\mathrm{InGaN} / \mathrm{GaN}$ LEDs on sapphire substrate which consists of three layers: a $3 \mu \mathrm{m}$ thick n-type $\mathrm{GaN}$ layer, five $2 \mathrm{~nm}$ thick QWs active layer and $0.2 \mu \mathrm{m}$ thick p-type $\mathrm{GaN}$ layer. The current density distributions were calculated in the drift approximation. The electrically neutral $\mathrm{p}$ - and n-type layers with constant electrical conductivity values were assumed. The active layer was considered as a layer with nonlinear conductivity represented by using the diode-like currentvoltage dependence $J=J_{s}\left(\exp \left(e V_{a} / \beta k_{B} T\right)-1\right)$, where $J$ is the vertical current density locally controlled by the voltage drop across the active layer $V_{a}, J_{s}$ is the saturation current density, $\beta$ is the $\mathrm{p}-\mathrm{n}$ junction ideality factor, $e$ is the elementary charge, $k_{B}$ is the Boltzmann constant and $T$ is temperature. Therefore, the electric potential and current density distributions follow from the equations $\vec{\nabla}\left(\sigma\left(x, y, z, V_{a}\right) \vec{\nabla} \varphi\right)=0$ and $\vec{J}=-\sigma\left(x, y, z, V_{a}\right) \vec{\nabla} \varphi$, where $\sigma\left(x, y, z, V_{a}\right)$ is the layer conductivity.

At the second stage of the simulation, the IQE can be calculated using the rate equation model and vertical current density distributions in the active layer $J(x, y)$ obtained at the first stage. As is commonly accepted, the IQE is defined as a ratio of the number of photons emitted from the active layer per second to the number of electrons injected into active layer per second [14]. The number of injected electrons may be determined by surface integrating of $J(x, y)$ over the whole area of the active layer $I / e=1 / e \iint J(x, y) \mathrm{d} S$, where $I$ is the total current. According to the rate equation, the relationship between the current density and injected carrier concentration under the steady state condition is given by

$$
\frac{J}{e d_{a}}=A n+B n^{2}+C n^{3}
$$

where $A, B$ and $C$ are the Shockley-Read-Hall (SRH), radiative and Auger coefficients, $n$ is the injected carrier concentration and $d_{a}$ is the thickness of the active layer which is assumed to equal total thickness of five QWs. From equation (1) one can numerically calculate lateral distributions of the injected carrier concentration $n(x, y)$ and the number of photons emitted from the active layer per second:

$$
\frac{P}{h v}=\mathrm{d}_{a} \iint B n^{2}(x, y) \mathrm{d} S
$$

where $P$ is the light output power, $h v$ is the photon energy.

Finally, the IQE is obtained as follows:

$$
\begin{aligned}
I Q E & =\frac{\mathrm{d}_{a} \iint B n^{2}(x, y) \mathrm{d} S}{1 / e \iint J(x, y) \mathrm{d} S} \\
& =\frac{\iint B n^{2}(x, y) \mathrm{d} S}{\iint\left(A n(x, y)+B n^{2}(x, y)+C n^{3}(x, y)\right) \mathrm{d} S} .
\end{aligned}
$$

Such a simple model of IQE calculation does not take into account either tunnelling or carrier leakage processes. Besides, we do not have any assumptions about carrier delocalization from In-rich regions in quantum wells.

\section{Temperature-dependent parameters}

The calculation of the efficiency droop temperature behaviour demands the knowledge of temperature dependences of the recombination coefficients and electrical conductivities. We use the values $B=2.0 \times 10^{-11} \mathrm{~cm}^{3} \mathrm{~s}^{-1}$ and $C=$ $2.0 \times 10^{-30} \mathrm{~cm}^{6} \mathrm{~s}^{-1}$ obtained from the photoluminescence measurements at room temperature [15]. The temperature dependence of $T^{-3 / 2}$ is assigned for radiative coefficient [14]. There is a lack of literature data about Auger coefficient in nitrides at low temperature. Nevertheless, we employ the activation temperature dependence $C(T)=$ $C_{o} \exp \left(-E_{C} / k T\right)$, where $C_{o}$ is a constant and $E_{C}$ the Auger activation energy. Constant $C_{o}$ is determined from the known Auger coefficient at room temperature. High room temperature $C$ value suggests the phonon-assisted type of the Auger process in nitrides rather than direct band-to-band process. The phonon-assisted process has weaker temperature dependence compared to the band-to-band process [16]; therefore, the activation energy $E_{C}$ in nitrides would be smaller than typical values obtained for the band-to-band process. Although the Auger activation energy in InGaN materials is still debatable, we employ $E_{C}=4.3 \mathrm{meV}$ based on the results presented in [17]. Reported room-temperature SRH coefficient values in InGaN are between $4.0 \times 10^{6} \mathrm{~s}^{-1}$ [18] and $2.0 \times 10^{8} \mathrm{~s}^{-1}$ [19]. In this paper SRH recombination was modelled assuming $A=3.0 \times 10^{7} \mathrm{~s}^{-1}$ at $300 \mathrm{~K}$ and using linear temperature decrease down to $2.0 \times 10^{7} \mathrm{~s}^{-1}$ at $150 \mathrm{~K}$ according to the experimental data [17] and theoretical calculations [20].

Electrical conductivity of the cladding layers is determined by the product of carrier concentration and mobility. The temperature-dependent electron and hole mobilities were represented by the Arora model with parameters for $\mathrm{GaN}$ [21]. The charge neutrality equation was used to obtain carrier concentration in the cladding layers at a given temperature. The donor concentration, acceptor concentration, degeneracy factor and activation energy of the impurity for $\mathrm{n}-\mathrm{GaN}(\mathrm{p}-\mathrm{GaN})$ cladding layer were chosen, respectively, as $N_{d}=1.0 \times 10^{20} \mathrm{~cm}^{-3}\left(1.2 \times 10^{19} \mathrm{~cm}^{-3}\right)$, $N_{a}=8.0 \times 10^{18} \mathrm{~cm}^{-3}\left(1.4 \times 10^{20} \mathrm{~cm}^{-3}\right), g=2$ (4), $E_{S i}=15 \mathrm{meV}$ [22] $\left(E_{M g}=152 \mathrm{meV}\right.$ [23] $)$. At room temperature these parameters give the carrier concentration of $5.0 \times 10^{18} \mathrm{~cm}^{-3}$ in $\mathrm{n}-\mathrm{GaN}$ and $5.2 \times 10^{17} \mathrm{~cm}^{-3}$ in the $\mathrm{p}-\mathrm{GaN}$ layer. Since the charge neutrality equation which takes into account single impurity level accurately describes the temperature dependence of carrier concentration at relatively high temperatures (see for example [23]), we have performed the simulation for $T>150 \mathrm{~K}$ only. The saturation current density and ideality factor need to be defined to include active layer conductivity in our simulation. Certain values for the saturation current density and ideality factor were extracted from the current-voltage measurements on blue InGaN LEDs (the room-temperature peak wavelength $\lambda=460 \mathrm{~nm}$ ) with 


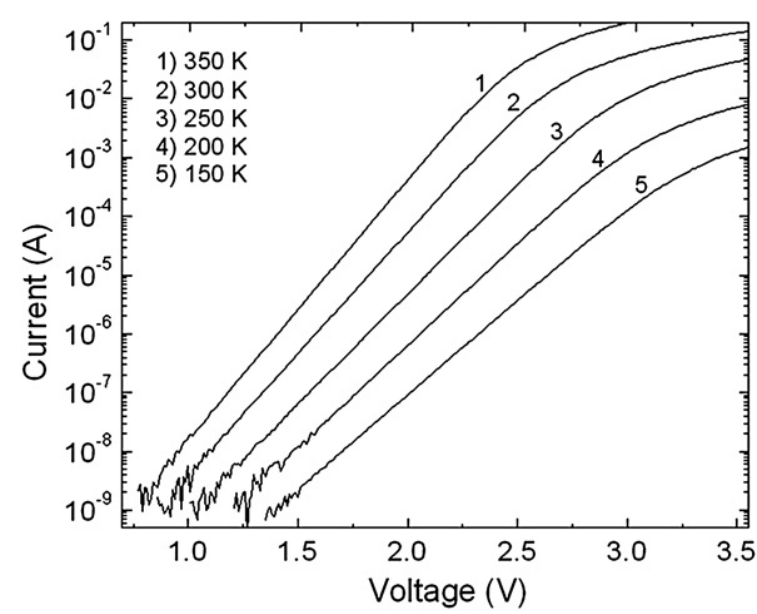

Figure 1. Current-voltage characteristics of blue $(\lambda=460 \mathrm{~nm})$ InGaN LED at various temperatures $(T=150-350 \mathrm{~K})$.

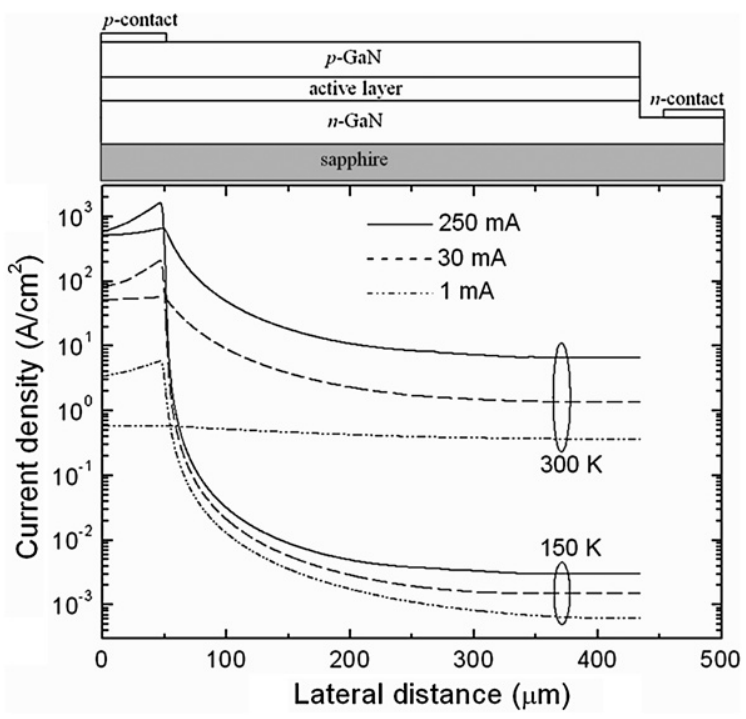

Figure 2. Lateral distributions of the current density in the LED active layer at $T=150 \mathrm{~K}$ and $300 \mathrm{~K}$.

the same structure as used in simulation (figure 1). Extracted ideality factor ranges form $\lambda \approx 2.2$ to 6 with temperature decreasing from $350 \mathrm{~K}$ to $150 \mathrm{~K}$.

\section{Results and discussion}

We analyse the current spreading in $0.5 \times 0.5 \mathrm{~mm}^{2}$ area InGaN LED on sapphire substrate with conventional bar-shaped contact geometry (figure 2). The results of simulations show that current spreading strongly depends on the temperature. In figure 2 the current density distributions in the active layer at 300 and $150 \mathrm{~K}$ are compared. At $300 \mathrm{~K}$, an increase of the applied bias is accompanied by essential localization of the current lines under the p-contact. The ratio of peakto-average current density grows up from 1.25 (practically uniform distribution) at $I=1 \mathrm{~mA}$ to 7 at $I=250 \mathrm{~mA}$. Contrary to that, at $150 \mathrm{~K}$ the ratio of peak-to-average current density reaches the value of 7 even at $I=0.6 \mathrm{~mA}$ and gradually increases up to 13 at $I=250 \mathrm{~mA}$. A strong $\mathrm{CC}$ effect at lower temperatures arises from increasing difference between

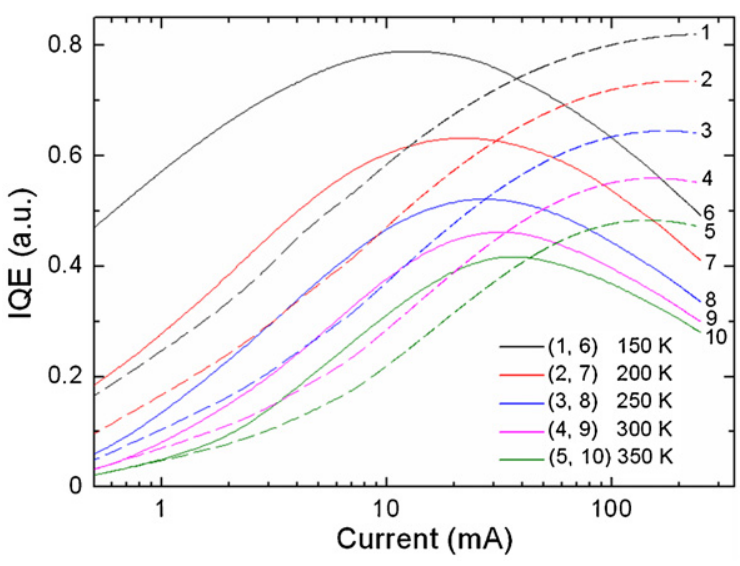

Figure 3. IQE versus current relations at various temperatures $(T=150-350 \mathrm{~K})$. Dashed curves (1-5)—one-dimensional rate equation calculations. Solid curves (6-10)—modified rate equation model that takes into account the $\mathrm{CC}$ effect.

$\mathrm{n}$ and $\mathrm{p}-\mathrm{GaN}$ layer conductivities. The electron mobility has its maximum value at about $T=150 \mathrm{~K}$, while the hole mobility peaks at $T=230 \mathrm{~K}$ and then begins to drop rapidly as temperature decreases due to impurity scattering in the highly $\mathrm{Mg}$-doped layer [23]. Besides, the high-Mg acceptor activation energy causes steeper low-temperature drop of the hole concentration compared to the electron concentration in the n-type layer. As a result, the lateral current path through n-type layer becomes even more preferable at lower temperatures and the current crowds more efficiently under the p-contact.

The current density distributions obtained at different applied biases were used to calculate the IQE. Figure 3 shows the results of calculations in two cases. The set of curves 1-5 represents the IQE of the InGaN LED without taking into account the nonuniform current density distributions. These curves were obtained based on the rate equation model in which the current density was considered to be spatially independent. In such one-dimensional approximation the IQE reaches its maximum when the current density is $J_{\max }=e d_{a} A(B+2 \sqrt{A C}) / C$ [24]. By using the assumed temperature dependences for the recombination coefficients, one can obtain that the $J_{\max }$ decreases from $120 \mathrm{~A} \mathrm{~cm}^{-2}$ at $150 \mathrm{~K}$ to $85 \mathrm{~A} \mathrm{~cm}^{-2}$ at $300 \mathrm{~K}$. This disagrees with the earlier reported experimental results. First, $J_{\max }$ values are much higher than commonly observed roll-off current densities in conventional InGaN LED. Second, $J_{\max }$ must increase with temperature [6]. However, due to specific structure of the LEDs designated to achieve maximum of the light extraction, the CC effect cannot be neglected in real devices and onedimensional simulations are not of value in principle. This takes place especially for InGaN LEDs on sapphire substrate with lateral injection geometry. As discussed above, the current density in the vicinity of the p-contact of the InGaN devices can be much higher than the average current density used in one-dimensional simulations. This leads to the high local carrier concentrations and activation of Auger recombination which results in drop of the IQE. The curves 6-10 in figure 3 were calculated by numerical integration of equations (1)-(3) taking into account the $\mathrm{CC}$ effect in 
the active layer. In this case, IQE versus current relations show reasonable roll-off current values which correspond to average current density in the range of $6-15 \mathrm{~A} \mathrm{~cm}^{-2}$. What is more important, the roll-off current value decreases and efficiency droop increases at lower temperatures. Since curves 6-10 in figure 3 were obtained using the same recombination coefficients as for one-dimensional calculations, such behaviour of IQE must be related only to the $\mathrm{CC}$ effect. From figure 2 it can be concluded that at the same injection levels the peak current density increases with decreasing of the temperature. For example, at $150 \mathrm{~K}$ the peak current density equal to $J_{\max }\left(120 \mathrm{~A} \mathrm{~cm}^{-2}\right)$ is reached when the current is $\approx 20 \mathrm{~mA}$ while at $300 \mathrm{~K}$ the $J_{\max }\left(85 \mathrm{~A} \mathrm{~cm}^{-2}\right)$ is reached at higher current $\approx 45 \mathrm{~mA}$. As a result, low-temperature IQE starts to drop at lower injection levels. The increase of the efficiency droop at low temperatures can be explained similarly. In the intermediate current range where the droop begins (10$40 \mathrm{~mA}$ ), the peak current density at $150 \mathrm{~K}$ is more than three times higher than that at $300 \mathrm{~K}$. Equation (1) yields that corresponding peak carrier concentration at $150 \mathrm{~K}$ is almost two times higher while the Auger coefficient is only $\sim 1.3$ times lower compared to the room-temperature value. Thus, the current crowding leads to the higher local Auger recombination rate at lower temperatures and higher droop effect. The degree of the efficiency droop defined as $\left(\operatorname{IQE}\left(I_{\text {peak }}\right)-\operatorname{IQE}\left(I_{250 \mathrm{~mA}}\right)\right) / \operatorname{IQE}\left(I_{\text {peak }}\right)$ is $40 \%$ and $32 \%$ for 150 and $300 \mathrm{~K}$ respectively.

Figure 4(a) compares the experimental InGaN LED efficiency as a function of current at several temperatures. In order to eliminate self-heating, the light-output versus current dependences were measured in the pulsed mode with $1 \%$ duty cycle and $1 \mathrm{kHz}$ frequency. The experimental efficiency versus current relations are in agreement with the numerical calculations presented in figure 3 at higher temperatures only. The increase of the overall experimental efficiency with temperature lowering is not as high as that predicted theoretically. In the range of $150-350 \mathrm{~K}$, the maximum of the experimental efficiency changes are $\sim 1.5$ times while the calculation shows $\sim 2$ times increase. To explain this discrepancy we took into account the phase-space filling (departure from the Boltzmann carrier statistics). Phasespace filling makes the radiative and Auger coefficients dependent on carrier concentration in the following forms: $B=B_{o} /\left(1+n / n_{c}\right)$ and $C=C_{o} /\left(1+n / n_{c}\right)$ [25], where $B_{o}$ and $C_{o}$ are the coefficients at low concentration $\left(n \ll n_{c}\right)$. We investigated the effect of phase-space filling on the IQE versus current relations by introducing the concentrationdependent recombination coefficients in equations (1)-(3). The solid curves in figure 4(a) were calculated by adjusting the parameter $n_{c}$ to fit the experimental data. The following values for $n_{c}$ were obtained: $2.2 \times 10^{18} \mathrm{~cm}^{-3}(150 \mathrm{~K})$, $5.0 \times 10^{18} \mathrm{~cm}^{-3}(200 \mathrm{~K}), \quad 9.6 \times 10^{18} \mathrm{~cm}^{-3}(250 \mathrm{~K})$, $2.1 \times 10^{19} \mathrm{~cm}^{-3}(300 \mathrm{~K}), 2.4 \times 10^{19} \mathrm{~cm}^{-3}(350 \mathrm{~K})$. Monotonic decrease of $n_{c}$ at temperature lowering is in agreement with fundamentals of carrier statistics. The lower the temperature, the lower the injection level at which the active layer of the LED becomes degenerate. Thus, at lower temperatures
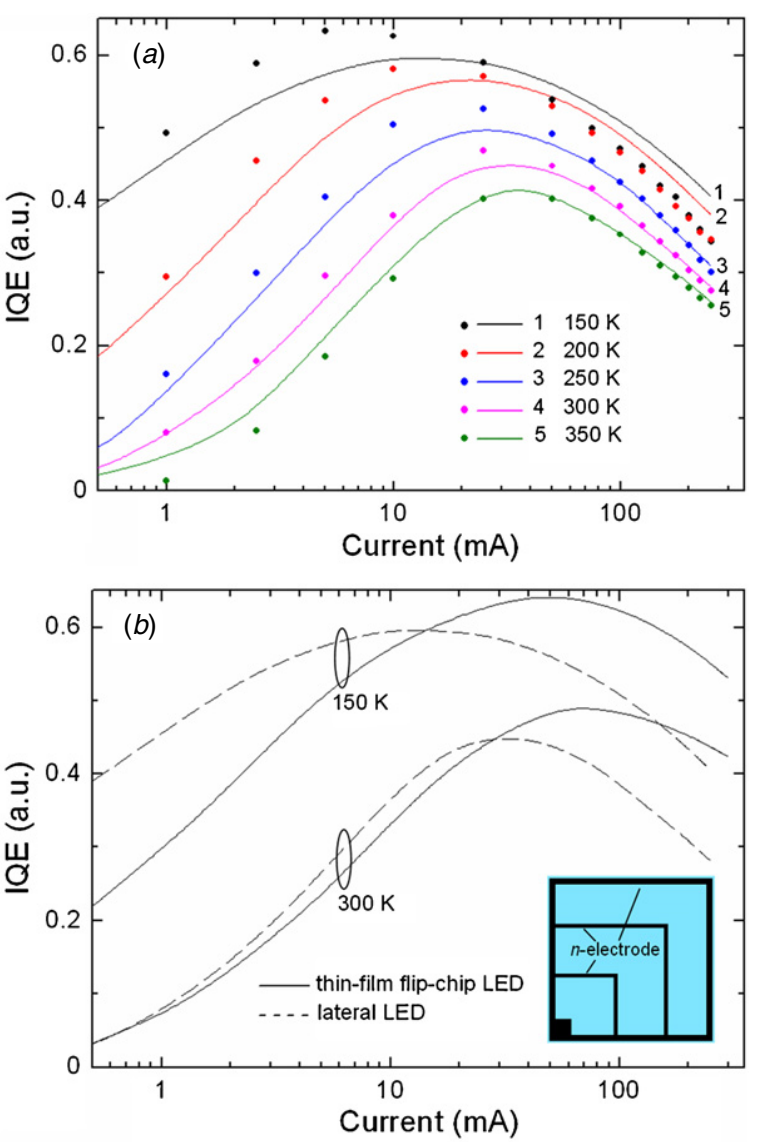

Figure 4. (a) Experimental efficiency versus current dependences for blue InGaN LED at various temperatures (symbols) and theoretical fits using the modified rate equation model with phase-space filling adjustments (solid curves). (b) Calculated IQE for lateral LED and vertical thin-film flip-chip LED with structured n-electrode.

a departure from the Boltzmann statistics occurs at lower current making the $n_{c}$ parameter temperature dependent. By comparing the calculated curves in figures 3 and $4(a)$, one can obtain that phase-space filling decreases the overall efficiency but does not change the IQE peak position. As a result, the misfit between the efficiency peak positions in the measured and fitted curves is still present at low temperatures. The reason for this deviation may be the longitudinal nonuniform carrier distribution in the MQW InGaN/GaN active layer. Poor hole transport through the GaN barriers makes the injected carriers recombine mainly in QW that lies closer to the p-layers [4]. Moreover, the nonuniformity appears to be more pronounced at lower temperatures due to the decrease of the hole mobility. Therefore, the 'recombination' thickness of the active layer is smaller than the physical thickness used in simulations and LED's efficiency is overestimated compared to experimental.

Finally, we discuss the efficiency droop in LEDs with vertical injection geometry. It is known that vertical LEDs show a more uniform current spreading compared with lateral LEDs [26]. Among vertical devices, thin-film flip-chip LEDs with structured n-electrode on the top seem to be the most promising due to utilizing the n-type GaN layer as a current spreading layer [27]. Figure 4(b) compares calculated IQE versus current for lateral and thin-film flip-chip LED at 150 and 
$300 \mathrm{~K}$. Above $I=30 \mathrm{~mA}$, thin-film flip-chip LED outperforms lateral LED at $300 \mathrm{~K}$. Moreover, thin-film flip-chip LED exhibits a lower shift of the roll-off current with decreasing temperature. All these results suggest that the efficiency droop can be remarkably reduced in devices with optimized current spreading.

\section{Conclusions}

We have investigated the temperature dependence of the efficiency droop in lateral InGaN/GaN MQW LEDs by using the modified rate equation model that takes into account the $\mathrm{CC}$ effect in the active layer of the devices. It was shown that the $\mathrm{CC}$ effect is more pronounced at lower temperatures making the local current density near the electrodes much higher than the average current density. Less uniform current density distribution leads to high local carrier concentration and the activation of Auger recombination which results in higher efficiency droop and lower roll-off current value at lower temperatures. Since we do not observe the droop by the 'CC free' one-dimensional modelling at reasonable injection levels, the temperature-induced shift of the IQE peak position to lower currents arises from the strong CC effect in the active region of InGaN LEDs. In addition, all presented results were obtained by using the simplest model based on the rate equation and commonly accepted values for recombination coefficients without any assumptions about carrier delocalization from In-rich regions and polarizationinduced carrier leakage out of quantum wells. Despite the fact that the modelled efficiency versus current relations do not exactly coincide with the experimental data, we show that lateral nonuniformity of the current density distribution results in the reduction of the IQE. Thus, the strategy of the InGaN LED's efficiency improvement and reduction of the efficiency droop would include not only optimization of the internal structures and parameters (energy band diagram, doping levels or thickness of quantum well/barrier) but also careful external device designing for minimization of the $\mathrm{CC}$ effect.

\section{References}

[1] Gardner N F, Müller G O, Shen Y C, Chen G, Watanabe S, Götz W and Krames M R 2007 Appl. Phys. Lett. 91243506
[2] Cao X A, Yang Y and Guo H 2008 J. Appl. Phys. 104093108

[3] Chichibu S F, Azuhata T, Sugiyama M, Kitamura T, Ishida Y, Okumura H, Nakanishi H, Sota T and Mukai T 2001 J. Vac. Sci. Technol. B 192177

[4] Xie J, Ni X, Fan Q, Shimada R, Özgür Ü and Morkoç H 2008 Appl. Phys. Lett. 93121107

[5] Kim M H, Schubert M F, Dai Q, Kim J K, Schubert E F, Piprek J and Park Y 2007 Appl. Phys. Lett. 91183507

[6] Laubsch A et al 2009 Phys. Status Solidi C 6 S913

[7] Yamane Y, Fujiwara K and Sheu J K 2007 Appl. Phys. Lett. 91073501

[8] Karpov S Y 2010 Phys. Status Solidi RRL 4320

[9] Shim J I, Kim H, Shin D S and Ryu H Y 2011 Proc. SPIE $793979391 \mathrm{~A}$

[10] Hammersley S, Badcock T J, Watson-Parris D, Godfrey M J, Dawson P, Kappers M J and Humphreys C J 2011 Phys. Status Solidi C 82194

[11] Kudryk Y Y and Zinovchuk A V 2011 Semicond. Sci. Technol. 26095007

[12] Ryu H Y and Shim J I 2011 Opt. Express 192886

[13] Guo X and Schubert E F 2001 J. Appl. Phys. 904191

[14] Schubert E F 2006 Light-Emitting Diodes 2nd ed (Cambridge: Cambridge University Press) p 86

[15] Shen Y C, Mueller G O, Watanabe S, Gardner N F, Munkholm A and Krames M R 2007 Appl. Phys. Lett. 91141101

[16] Kioupakis E, Rinke P, Delaney K T and Van de Walle C G 2011 Appl. Phys. Lett. 98161107

[17] Jang D J, Lin G T, Hsiao C L, Tu L W and Lee M E 2008 Appl. Phys. Lett. 92042101

[18] David A and Grundmann M J 2010 Appl. Phys. Lett. 97033501

[19] Dai Q et al 2009 Appl. Phys. Lett. 94111109

[20] Karpov S Y and Makarov Y N 2002 Appl. Phys. Lett. 814721

[21] Piprek J 2007 Nitride Semiconductor Devices: Principles and Simulation (Berlin: Wiley-VCH) p 78

[22] Götz W, Johnson N M, Chen C, Liu H, Kuo C and Imler W 1996 Appl. Phys. Lett. 683144

[23] Kozodoy P, Xing H, DenBaars S P, Mishra U K, Saxler A, Perrin R, Elhamri S and Mitchel W C 2000 J. Appl. Phys. 871832

[24] Ryu H Y, Kim H S and Shim J I 2009 Appl. Phys. Lett. 95081114

[25] David A and Grundmann M J 2010 Appl. Phys. Lett. 96103504

[26] Kim H, Kim K-K, Choi K-K, Kim H, Song J-O, Cho J, Baik K H, Sone C, Park Y and Seong T-Y 2007 Appl. Phys. Lett. 91023510

[27] Bogdanov M V, Bulashevich K A, Khokhlev O V, Evstratov I Y, Ramm M S and Karpov S Y 2010 Phys. Status Solidi C 72124 